\title{
AN ANALYSIS OF THE PARTIAL WAVE EXPANSION OF THE DISPERSION ENERGY FOR Ne ${ }_{2}$ *
}

\author{
G. CHALASINSKI \\ Deparment of Chemistry, University of W'arsaw, Pasteura 1.02-093 W'arsaw. Poland \\ and The Theoretical Chemistry. Group. Liniversity of Utrecht. Padualan 8. De Uithof. Ctrecht. The Netherlands \\ and \\ J.H. VAN LENTHE and Th.P. GROEN ** \\ The Theoretical Chemistry Ciroup. Ciniversity of Utrecht, Padualaan S. De Cithof. Utrecht. The . Vetherlands
}

Received 1 June 1984: in final form 10 July 1984

\begin{abstract}
Calculations of the dispersion energy for $\mathrm{Ne}_{2}$ by using the partial wave expansion through the h-h term were carriced out in the region of the van der thals minimum. It is shown that, at $k=60_{0}$. lack of higher than $\mathrm{d}$ orbitals gives a dispersion energy in error by $\approx 16 \%$, and lack or higher than $f$ orbinals gives an error of $\approx 6 \%$.
\end{abstract}

\section{Introduction}

Recently, in his review article on intermolecular in?teractions. Scoles concluded that "intermolecular forces have entered the age of spectroscopic precision" [1]. In particular, this is the case for the raregas dimers, where precise potentials reproducing accurately a variety of dilute-gas properties have recently been published $\left(\mathrm{He}_{2}[2] . \mathrm{Ne}_{2}[3], \mathrm{Ar}_{2}\right.$ [4.5]). Consequently, more than ever before, in order that ab initio calculations be useful they' should be of high quality and should give special attention to defining what has been left out of the calculations.

$\mathrm{Ab}$ initio calculations of the non-expanded dispersion contribution to the interaction energy rarely take into account higher than d polarization functions. To the best of our knowledge. $f$ functions were used (except for small model systems. $\mathrm{He}_{2}[6.7]$ and $\mathrm{Be}_{2}$ [8]) only for the Ne dimer [9] and $\mathrm{Ar}-\mathrm{HCl}$ [10], whereas higher than $f$ functions have never been used.

* This work was partly supported by the Polish Academy of Sciences within the project MR.I.9.

** Present address: Control Data, J.C. van Markenlaan 5, 2285 VL Rijswijk, $Z$ H. The Netherlands.
In many cases, when a $10: 0$ or larger error in the interaction energy is acceptable. this limitation does not seem to be serious. In addition. since the interaction potential is the net result of a balance of repulsive and attractive contributions. quite often fortuitous cancellation of errors may occur and obscure the actual role of higher polarization functions. For instance, a simple $a b$ init io approximation of the interaction energy as the sum of the SCF interaction energy and the dispersion cont ribution (SCF-DISP) $[11,12]$ may work quite well for rare-gas dimers wi1hout higher polarization functions $[9,13.14]$. This is due to cancellation of the intra-atomic correlation correction to the first-order exchange energy (repulsive) and the interaction of higher instantaneous multipoles (attractive) $[13,9]$. Both these effects are disregarded in the SCF-DISP approximation.

The aim of this paper is to elucidate the role of higher polarization functions in calculations of the dispersion energy for a model rare-gas dimer, $\mathrm{Ne}_{2}$, in the region of the van der Waals minimum. We use the partial wave expansion as described in ref. [9] which is evaluated through the $\mathrm{h}-\mathrm{h}$ term. We believe that the results presented here will provide a better understanding of the attractive part of the Ne-Ne poten- 
tial. They also shed new light on the reliability of limited-basis-set calculations of the dispersion effect by means of both the perturbation and supermolecule variational methods.

\section{Theory}

The dispersion energy between two spherically symmetric systems A and B may be written in the form of the partial wave expansion [9]

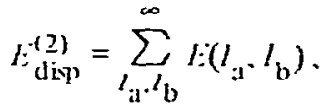

$$
\begin{aligned}
& l:\left(l_{\mathrm{a} a} \cdot l_{\mathrm{b}}\right)=\sum_{i_{\mathrm{a} \cdot} j_{\mathrm{b}}}^{a c c} \sum_{m=-1<}^{1<} t\left(i_{\mathrm{a} 1} \rightarrow l_{\mathrm{a} \cdot}, j_{\mathrm{b}} \rightarrow l_{\mathrm{b}}, m\right) .
\end{aligned}
$$

where $i_{i t}\left(j_{h}\right)$ denote the sets of quantum numbers

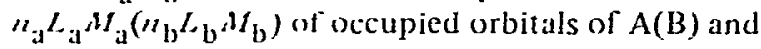
$I_{\mathrm{a}} \cdot I_{\mathrm{b}}$ are angular quantum numbers. Explicit expressions for pair dispersion energies $l:\left(i_{\mathrm{a}} \rightarrow l_{\mathrm{a}}, j_{\mathrm{b}}-l_{\mathrm{b}}, m\right)$ were given in ref. [9]. For two Ne atoms, $L_{\mathrm{i} 1}, L_{\mathrm{b}} \leqslant 1$ and the multipole expansion of $l:\left(i_{a}-l_{a} . j_{b} \rightarrow l_{b}, m\right)$ is a polynomial in $R^{-1}$. the asymptotically leading term of which may be found from the formula [9]

$$
l:\left(n_{\mathrm{a}} L_{\mathrm{a}} M_{\mathrm{a}} \rightarrow l_{\mathrm{a}}, n_{\mathrm{b}} L_{\mathrm{b}} M_{\mathrm{b}}-l_{\mathrm{b}}, m\right) R^{-2\left(k_{\mathrm{a}}+k_{\mathrm{b}}+1\right)} \text {. }
$$

where

$$
\begin{aligned}
k_{x} & =l_{x} & & \text { for } L_{x}=0 ; \\
& =l_{x}+2 \delta_{0, l_{x}}+2 \delta_{1, l_{x}}-1, & & \text { for } L_{x}=1 .
\end{aligned}
$$

Knowledge of (3) is necessary to reorganize the partial wave expansion in such a way that subsequent terms are varying asymptotically as subsequent even powers of $R^{-1}[9]$ :

$l_{\mathrm{d} i \mathrm{w}}^{(2)}=\sum_{n=0,8,10 \ldots} l:(n)$.

Where

$l:(n)=\sum_{i_{\mathrm{a}}, j_{\mathrm{b}}}^{\operatorname{acc}} \sum_{l_{\mathrm{a}}, l_{\mathrm{b}}}^{2\left(k_{\mathrm{a}}+k_{\mathrm{h}}+1\right)=n} l:\left(i_{\mathrm{a}}-l_{\mathrm{a}} \cdot j_{\mathrm{b}} \rightarrow l_{\mathrm{b}}\right)$

and $k_{\mathrm{a}}$ and $k_{\mathrm{b}}$ are given by (3).

Since. in the region of the van der Waals minimum, pair dispersion interactions including the $2 \mathrm{~s}$ orbital are an order of magnitude smaller than the $2 \mathrm{p}_{\mathrm{a}}-2 \mathrm{p}_{\mathrm{b}}$ interactions, it is expedient here to use a different partitioning of $E_{\text {disp }}^{(2)}$ :

$E_{\mathrm{disp}}^{(2)}=\sum_{n=6,8.10, \ldots}^{\infty} \widetilde{E}(n)$.

$\widetilde{E}(n)=\sum_{l_{\mathrm{a}}, l_{\mathrm{b}}} E\left(l_{\mathrm{a}}, l_{\mathrm{b}}\right)$.

$2\left(l_{\mathrm{a}}+l_{\mathrm{b}}+2 \delta_{0, l_{\mathrm{a}}}+2 \delta_{1, l_{\mathrm{a}}}+2 \delta_{0, l_{\mathrm{b}}}+2 \delta_{1, l_{\mathrm{b}}}-1\right)=n .(7)$

The sum in eq. (7) is over the $l_{\mathrm{a}}, l_{\mathrm{b}}$ for which $I\left(2 \mathrm{P}_{\mathrm{a}} \rightarrow l_{\mathrm{a}}\right.$. $2 \mathrm{p}_{\mathrm{b}} \rightarrow \bar{I}_{\mathrm{b}}$ ) varies asymptotically as $R^{-n}$, i.e. as if the asymptotic behaviour of $l:\left(l_{\mathrm{a}}, l_{\mathrm{b}}\right)$ were determined by the $2 \mathrm{p}_{a}-2 \mathrm{p}_{\mathrm{b}}$ dispersion interaction. This is not true only if $l_{\mathrm{a}}$ and/or $l_{\mathrm{b}}=1$. In this case, $\ell\left(i_{\mathrm{a}}-l_{\mathrm{a}}, j_{\mathrm{b}} \rightarrow l_{\mathrm{b}}\right)$ for $i_{\mathrm{a}}$ and $/$ or $i_{\mathrm{b}}=2 \mathrm{~s}$ provide the asymptotically leading contributions. This inconsistency will become serious only at larger distances. since $\widetilde{E}(8)$ and $\widetilde{E}(10)$ include terms of the order of $R^{-6}$. For $n>10, E(n)=\widetilde{L}(n)$.

\section{Analysis of the partial wave expansion of $E_{\text {diøp }}^{(2)}$ for $\mathrm{Ne}_{2}$}

By using the method described in ref. [9], the variant with the Epstein-Nesbet zero-order Hamiltonian, the values of $\ell\left(n L_{\mathrm{at}} \rightarrow l_{\mathrm{a}}, n L_{\mathrm{b}} \rightarrow l_{\mathrm{b}}\right)$ have been calculated for $R=5.0,5.5,6.0$ and $7.0 a_{0}, n L_{\mathrm{a}}, n L_{\mathrm{b}}$ $=1 \mathrm{~s}, 2 \mathrm{~s}$ and $2 \mathrm{p}$, and $l_{\mathrm{a}}, l_{\mathrm{v}}=0,1,2,3,4$ and 5 . Throughout the calculations, Gaussian orbitals with spherical harmonics for angular parts were used. The occupied orbitals were represented as in ref. [9]. The basis set used to expand the dispersion function is displayed in table 1 . The $A T M O L$ integral package [15] and INTACT [16] were used to calculate integrals and dispersion energies, respectively.

The values of individual $E\left(I_{a}, I_{b}\right)$ and $\widetilde{E}(n)$ are shown in table ?. All $E(n)$ for $n \leqslant 12$ are complete. For $n>12$, the following terms are missing: $i-d$ for $n=14: \mathrm{i}-\mathrm{f}, \mathrm{i}-\mathrm{p}$ and $\mathrm{j}-\mathrm{d}$ for $n=16 ; \mathrm{i}-\mathrm{g}, \mathrm{j}-\mathrm{f}, \mathrm{j}-\mathrm{p}$ and $k-d$ for $n=18$. The asymptotically leading interactions of instantaneous multipoles to which a particular $E\left(I_{\mathrm{a}} \cdot I_{\mathrm{b}}\right)$ (strictly speaking $E\left(2 \mathrm{p}_{\mathrm{a}} \rightarrow I_{\mathrm{a}}, 2 \mathrm{p}_{\mathrm{b}}-I_{\mathrm{b}}\right)$, cf. section 2) may be related are also indicated.

Table 2 does not contain explicitly terms including s orbitals. They are always allowed for together with 
Table 1

The exponents of GTOs used to expand the dispersion function

\begin{tabular}{|c|c|c|c|}
\hline \multirow{2}{*}{ Type of GTO } & \multicolumn{3}{|c|}{ Exponents $\left(a_{0}^{-2}\right)$} \\
\hline & 0.15 & 0.30 & 0.06 \\
\hline $2 p^{a)}$ & 0.1 & 0.3088 & 0.70 .4 \\
\hline $3 d$ a) & 1.12 & 0.32 & \\
\hline $41^{-b)}$ & 0.9 & 0.225 & \\
\hline $5 \mathrm{~g} c)$ & 0.2 & & \\
\hline $6 \mathrm{~h}$ d) & 0.17 & & \\
\hline
\end{tabular}

a) The same as in ref. [9].

b) Chosen so as to maximize the value of $E\left(2 p_{a} \rightarrow d_{a} \cdot 2 p_{b}-f_{b}\right)$.

c) Chosen so as to maximize the value of $E\left(2 \mathrm{p}_{\mathrm{a}}-\mathrm{d}_{\mathrm{a}} .2 \mathrm{p}_{\mathrm{b}}-\mathrm{g}_{\mathrm{b}}\right)$.

d) Chosen so as to maximize the value of $E\left(2 \mathrm{p}_{\mathrm{a}} \rightarrow \mathrm{d}_{\mathrm{a}}, 2 \mathrm{p}_{\mathrm{b}}-\mathrm{h}_{\mathrm{b}}\right)$. d orbitals (which are narked by a tilde). They musi not be neglected, but it has been found that they are very well reproduced solely by the spherical combinations of Cartesian Gaussians [9] ${ }^{*}$. In this work, since we have used Gaussians multiplied by spherical harmonics, separate s orbitals had to be added.

It is important to estimate the accuracy of the calculation of individ $\lrcorner$ al terms. It has previousl $y$ been found [9] and confined heas :iat two polarization

\footnotetext{
In ref. [9], where two spherical combinations of d orbitals were present in the basis set, additional s orbitals gave negligible contributions. This las led to the misleading comment that if $l_{\mathrm{a}}$ or $l_{\mathrm{b}}=0$, then $E\left(l_{\mathrm{a}}, l_{\mathrm{b}}\right)$ is negligible.
}

Table ?

Individual partial wave components $E\left(l_{\mathrm{a}}, l_{\mathrm{h}}\right)$ and the total dispersion energy. $\widetilde{\mathrm{d}}$ stands for $\mathrm{s}+\mathrm{d} . R$ in $a_{0}$ - energies in $10^{-5}$ hartree

\begin{tabular}{|c|c|c|c|c|c|c|}
\hline \multirow[t]{2}{*}{$l_{\mathrm{a}}-l_{\mathrm{b}}$} & \multirow{2}{*}{\multicolumn{2}{|c|}{$\begin{array}{l}\text { Multipole } \\
\text { interaction }\end{array}$}} & \multicolumn{4}{|l|}{$E\left(I_{\mathrm{a}} \cdot l_{\mathrm{b}}\right)$} \\
\hline & & & $R=5.0$ & $R=5.5$ & $R=6.0$ & $R=7.0$ \\
\hline$\overline{\mathrm{d}}-\tilde{\mathrm{d}}$ & $E(6)$ & $=\mathrm{DD}$ & 41.9494 & 23.5871 & 13.8365 & 5.3700 \\
\hline \multirow[t]{2}{*}{$2 \times(\mathrm{d}-\mathrm{n})$} & & $\begin{array}{l}\mathrm{DQ} \\
\mathrm{DQ}\end{array}$ & $\begin{array}{r}8.0054 \\
11.4687\end{array}$ & $\begin{array}{l}4.1298 \\
5.5861\end{array}$ & $\begin{array}{l}2.2354 \\
2.8056\end{array}$ & $\begin{array}{l}0.7453 \\
0.7961\end{array}$ \\
\hline & $E(8)$ & $=$ & 19.4741 & 9.7159 & $5.0+10$ & $1.5+14$ \\
\hline $\begin{array}{r}p-p \\
2 \times(f-p) \\
f-f^{\prime} \\
2 \times(g-\tilde{d})\end{array}$ & & $\begin{array}{l}\mathrm{QQ} \\
\mathrm{QQ} \\
\mathrm{QQ} \\
\mathrm{OD}\end{array}$ & $\begin{array}{l}0.5984 \\
1.5609 \\
1.2898 \\
2.6881\end{array}$ & $\begin{array}{l}0.2510 \\
0.6864 \\
0.5738 \\
1.2708\end{array}$ & $\begin{array}{l}0.1165 \\
0.3190 \\
0.2589 \\
0.5911\end{array}$ & $\begin{array}{l}0.0317 \\
0.0785 \\
0.0567 \\
0.1319\end{array}$ \\
\hline \multicolumn{3}{|c|}{$E(10)=$} & 6.1372 & 2.7820 & 1.2805 & 0.2988 \\
\hline $\begin{array}{l}2 \times(g-p) \\
2 \times(g-f) \\
2 \times(h-d)\end{array}$ & & $\begin{array}{l}\text { OQ } \\
\text { OQ } \\
\text { HD }\end{array}$ & $\begin{array}{l}0.4848 \\
0.6792 \\
0.8567\end{array}$ & $\begin{array}{l}0.1955 \\
0.3207 \\
0.4133\end{array}$ & $\begin{array}{l}0.0833 \\
0.1460 \\
0.1901\end{array}$ & $\begin{array}{l}0.0166 \\
0.0288 \\
0.0375\end{array}$ \\
\hline \multicolumn{3}{|c|}{$E(12)=$} & 2.0207 & 0.9295 & 0.4194 & 0.0829 \\
\hline $\begin{array}{c}\mathrm{g}-\mathrm{g} \\
2 \times(\mathrm{h}-\mathrm{p}) \\
2 \times(h-f)\end{array}$ & & $\begin{array}{l}\text { OO } \\
\text { HQ } \\
\text { HO }\end{array}$ & $\begin{array}{l}0.0558 \\
0.2287 \\
0.2305\end{array}$ & $\begin{array}{l}0.0347 \\
0.0846 \\
0.1159\end{array}$ & $\begin{array}{l}0.0191 \\
0.0328 \\
0.0544\end{array}$ & $\begin{array}{l}0.0044 \\
0.0056 \\
0.0106\end{array}$ \\
\hline & \multicolumn{2}{|c|}{$E(14)=$} & 0.5150 & 0.2352 & 0.1063 & 0.0206 \\
\hline $\begin{array}{c}2 \times(h-g) \\
h-h\end{array}$ & \multicolumn{2}{|c|}{$\begin{array}{l}E(16): \mathrm{HO} \\
E(18): \mathrm{HH}\end{array}$} & $\begin{array}{l}0.0267 \\
0.0030\end{array}$ & $\begin{array}{l}0.0189 \\
0.0024\end{array}$ & $\begin{array}{l}0.0118 \\
0.0017\end{array}$ & $\begin{array}{l}0.0033 \\
0.0006\end{array}$ \\
\hline \multicolumn{3}{|l|}{ sum } & 70.1261 & 37.2710 & 20.7022 & 7.3176 \\
\hline
\end{tabular}

a) $\mathrm{D}=$ dipole, $\mathrm{Q}=$ quadrupole, $\mathrm{O}=$ octupole, $\mathrm{H}=$ hexadecapole. 
funclions for a given symmetry, with optimized exponents, reproduce a particular term with $\approx 1 \%$ error. Calculations including only one polarization function of a given symmetry are less accurate. For example, use of only one orbital to calculate the $d-f$ and $f-f$ tcrms yields respectively $92 \%$ and $87 \%$, of the result obtained with two forbitals. Exactly the same conclusion was found to be valid for the $d-g$ and $g-g$ temis. The above remarks allow us to est imate the enur in $l_{\text {digr }}^{(2)}$ due to the fact that only one $g$ and one h function was used. The result ing error in the lotal $l_{\mathrm{diip}}^{(2)}$ is ranging from $0.7 \%$ at $R=5 a_{0}$ to $0.3 \%$ at $\mu=7 a_{0}$.

The values of $1 \frac{(2)}{\text { disp }}$ in table 2 are also in error because the partial wave expansion has been truncated and includes only terms for $l_{\mathrm{a}}, l_{\mathrm{b}}<6$. One can see that the series (6) is well convergent and the langest of the missing terms, the $\mathrm{d}-\mathrm{i}$ term, corresponds to dipule $-2^{5}$-pule is'pe and belongs to $\widetilde{t}(14)$.

Since dipole-ociupole and dipole-hexadecapole tems provide $=45 \%$ of $\widetilde{I}(10)$ and $\widetilde{I}(12)$. respectively (independent of $R$ ), it is reasomable to assume that the dipole $2^{5}$-pole term yields also about half of the (1)tal $l:(14)$. This assumption and perusal of the $\mathrm{h}-\mathrm{g}$ and $h-h$ terms leads to the conclusion that higher than hobitals would give a contribution approximately equal to $0.7 \%$ at $R=5 a_{0}$ and $0.2 \%$ at $R=7 a_{0}$ of $l_{\text {dip }}^{-15}$

From the ahove discussion of the errors of individual $l\left(l_{t}, i_{1}\right)$ and of the error of truncation of the series (6), one may conclude that, as far as basis-set limitations are concerned, the reported values of $E_{\mathrm{dit}}^{(2)}$ may be in error by $1.5 \%$ at $R=5 a_{0} 100.5 \%$ at $R=7 a_{0}$.

Finally. we should comment on the error which results from the indequacy of the method of calcu. lation of the dispersion energy. Since the procedure is based on the Epstein- Nesbet zero-order Hamiltonian ("statc-energy denominators") [17]. not only is the intra-atomic correlation neglected but the definition of the procedure itself reveals certain drawbacks $[18.9]$. On the other hand, the procedure gives van der Waals coefficients for He 2 close to the experimental ones [18], and this would also seem to be valid for $\mathrm{Ne}_{2}[9]$. Nevertheless, since the van der Waals coefficients for $\mathrm{Ne}_{2}$ are not known with sufficient accuracy [19], we are unable to analyse this error.

From the point of view of supermolecule calculations of the dispersion effects. it is important to know what accuracy can be achieved if a basis set consists of orbitals up to a given angular quantum number. Assuming that the total result in table 2 is exact, one can find that inclusion of only up to $d$. up to f and up to $g$ orbitals provides (in the range $5.0-7.0 a_{0}$ ) $72.1 \%$ to $84.0 \% .92 .5 \%$ to $96.7 \%$, and $98.1 \%$ to $99.2 \%$, respectively, of $l:$ dipp. At the van der Waals minimum, the error in the interaction energy may be substantially larger. as the repulsive contribution cancels about half of the dispersion effect. For instance. lack of higher than f functions. assuming that no fortuitous cancellation of errors occurs, may in approximately $10 \%$ and $25 \%$ error in the interaction energy at $R=6 a_{0}$ and $5.5 a_{0}$. respectively. Unfortunately. since one cannor use the partial wave expansion within the supermolecule approach, a basis set should include all necessary polarization finctions at the same time and hence would rapidly become prohibitively large. At present the only remedy is to carry out so-called "balanced" calculations. i.c. based on the more-or-less precise knowledge of possible cancellations of different errors. both basis set and conceptual. An example of such a method is the ICF (interaction correjated fragments) method of Lit and Mc Lean [20].

\section{Interaction potential for $\mathrm{Ne}_{2}$}

Recently, a few accurate semi-empirical potentials for $\mathrm{Ne}_{2}$ have been proposed and proved to be very successful in reproducing a variety of dilute-gas properties of $\mathrm{Ne}$ (cf. ref. [3] and references therein). l'articularly interesting from the point of view of comparison with ab initio perturbation calculations is the so-called exchange Coulomb (XC) potential model. the antractive long-range part $\Delta E_{c}$ of which consists of a sum of the first three terms of the multipole expansion for the second-order dispersion energy multiplied by a proper damping function:

$\Delta E_{c}=-\left(C_{6} / R^{6}+C_{8} / R^{8}+C_{10} / R^{10}\right) f(R)$.

The values of individual $C_{l l}$, in particular if they are allowed to vary within the bounds of Tang et al. [19], may be only approximately compared with calculated ab initio values. On the other hand, the total $\Delta E_{c}$ effect is expected to model accurately the net attractive effect. According to symmetry-adapted perturbation theory, $\Delta E_{c}$ accounts for the second-and higher-order 
Table 3

Interaction entergy (in $10^{-5}$ hartree) and its components between Ne atoms, from ab initio and semi-cmpirieal resutrs

\begin{tabular}{|c|c|c|c|c|}
\hline & \multicolumn{4}{|l|}{$R\left(a_{0}\right)$} \\
\hline & 5.0 & 5.5 & 6.0 & 7.0 \\
\hline$د E_{\mathrm{SCF}}^{\mathrm{int}}$ & 74.01 & 21.76 & 6.38 & 0.54 \\
\hline$E_{\text {disp }}^{(\mathrm{P})}$ & -70.13 & -37.27 & -20.70 & -7.32 \\
\hline$E_{\text {ex }-\operatorname{disp}}$ & 3.92 & 1.22 & 0.38 & 0.04 \\
\hline$E \operatorname{disp}^{(2)}+E_{\mathrm{ex}-\mathrm{disp}}$ & -66.21 & -36.05 & -20.32 & -7.27 \\
\hline$\Delta E_{\mathrm{SCl}}^{\mathrm{int}}+E_{\mathrm{disp}}^{(2)}+E_{\mathrm{ex}-\mathrm{disp}}$ & 7.80 & 14.29 & -13.95 & -6.74 \\
\hline$E_{\mathrm{Sc}}(\mathrm{XC}-2)^{\mathrm{b})}$ & 84.98 & 25.26 & 7.51 & 0.66 \\
\hline$\Delta E_{\mathrm{C}}{ }^{\mathrm{b})}$ & -66.37 & -35.68 & -20.24 & -7.44 \\
\hline$E_{\text {in }}\left(x(-2)^{b)}\right.$ & 18.61 & -10.42 & -12.73 & -6.78 \\
\hline$E_{\mathrm{SR}}(\mathrm{XC}-2)+E_{\text {disp }}^{(2)}+E_{\mathrm{ex}-\mathrm{disp}}$ & 18.77 & -10.79 & -12.81 & -6.61 \\
\hline
\end{tabular}

a) from ref. 19]. b) Colculated from rel. [3].

dispersion energies damped by overlap and exchange dispersion effects [9]. We have compared in table 3 the values of $\Delta E_{\mathrm{e}}$ for the best of the XC potentials, $\mathrm{XC}-2$, with the values of $E_{\text {dign }}^{(2)}$ from this work quenched by the exchange dispersion effect. $E_{\text {ex-disp, }}$ calculated in ref. [9]. One can see a remarkably good agreement between $E_{\text {disp }}^{(2)}$ and $\Delta E_{\mathrm{c}}$. We should keep in mind here that an approximate algorithm for $E_{\text {disp }}^{(2)}$ has been used and that we neglected the third-order dispersion contribution which must be accounted for by $\Delta E_{\mathrm{c}}$. On the other hand, the semi-empirical character of the XC-2 potential does not permit its precise division into well-defined terms. Consequently, a detailed analysis of a small discrepancy between $\Delta E_{\mathrm{c}}$ and $E_{\mathrm{disp}}^{(2)}$ would not be meaningful.

We have also calculated the total interaction energy in the SCF-DISP approximation modified by adding $E_{\text {cx-disp }}$ [9]:

$E_{\mathrm{int}} \approx \Delta E_{\mathrm{SCF}}^{\mathrm{int}}+E_{\mathrm{disp}}^{(2)}+E_{\mathrm{ex}-\mathrm{disp}}$

where $\triangle E_{\mathrm{SCF}}^{\text {int }}$ is the SCF interaction energy.

It is seen from table 3 that eq. (9) provides too deep a curve and even the position of the minimum is shifted to shorter $R$. The conclusion is that $\Delta E_{\mathrm{SCF}}^{\mathrm{int}}$ underestimates the repulsive part of the $\mathrm{Ne}_{2}$ potential. Indeed, if one replaces $\Delta E_{\mathrm{SCF}}^{\mathrm{int}}$ in eq. (7) by the short-range part of the XC-2 potential, $E_{\mathrm{SR}}(\mathrm{XC}-2)$, the values of the interaction energy are very close to those of the XC-2 potential (cf table 3 ).

On the basis of the results for the He dimer [13.2]. one can expect that the difference $F_{\mathrm{SR}}(\mathrm{XC}-2)-\Delta E_{\mathrm{SCI}}^{\mathrm{int}}$ is manly due to the intra-atomic correlation correction to the first-order interaction energy. If so, this contribution would be far from negligible.

\section{Acknowledgement}

One of us (GC) is very grateful to F.B. van Duijneveldt for his kind hospitality during his stay at the Rijksuniversiteit Utrecht. M. Bulski is acknowledged for reading and commenting on the manuscript.

\section{References}

[1] G. Scoles, Ann. Rev, Phys. Chem. 31 (1980) 81.

[2] R. Feltgen, H. Kirst. K.A. Köhler. II. Pauly and l- Torello, J. Chem. Phys. 76 (1982) 2360.

[3] R.A. Aziz, W.J. Meath and A.R. Alnatt, Chem. Phy's. 78 (1983) 295.

14] A. Koide, W.J. Meath and A.R. Allnatt. Mol. Phys 39 (1980) 895.

[5] R.A. Aziz and M.H. Chen. J. Chem. Phys. 67 (1978) 5719. 
[6] G. Chatasiński, B. Jeziorski, K. Szalewicz and J. Andzelm, Mfol. Phys. 33 (1977) 971.

(7) H.F. Schacfer III and D.R. McLaughlin. Chem. Phys. Letters 12 (1971) 244.

[8] B.H. Lengsfield III, A.D. McLean, M. Yoshimine and B. Liu. J. Chem. Phy's. 79 (1983) 1891.

19] G. Chałasinski. Mol. Phys. 49 (1983) 1353.

(10) T. van Dam. Ph.D. Thesis. University of Utrecht (1984).

[11] E. Kochanski, Theoret. Chim. Acta 39 (1975) 339.

[12] B. Jeziurski and M.C. van Hemert, Mol. Phys. 3 1 (1976) 713.

[13] G. Chalasinski, S. van Smaalen and F.B. van Duijneveldt. Mol. Phys, 45 (1982) 1113
[14] J. Andzelm, S. Huzinaga, M. Klobukowski and E. Radzio. to be published.

[15] V.R. Saunders. ATMOL4, Molecular integrals, (1979).

[16] Th.P. Groen, INTACT, Internal Report, Utrecht (1975).

[17] E. Kochanski, J. Chem. Phys. 58 (1973) 5823.

[18] F. Mulder, Ph.D. Thesis, University of Nijmegen, Nijmegen (1978).

[19] K.T. Tang, J.M. Norbcek and P.R. Certain, J. Chem. Phys. 64 (1976) 3063.

[20] B. Liu and A.D. McLean, J. Chem. Phys. 72 (1980) 3418. 